2017-12-27

\title{
Epistaxis 2016: national audit of
} management

\section{INTEGRATE,}

http://hdl.handle.net/10026.1/10618

10.1017/S002221511700202X

Journal of Laryngology and Otology

Cambridge University Press (CUP)

All content in PEARL is protected by copyright law. Author manuscripts are made available in accordance with publisher policies. Please cite only the published version using the details provided on the item record or document. In the absence of an open licence (e.g. Creative Commons), permissions for further reuse of content should be sought from the publisher or author. 


\title{
EPISTAXIS 2016: NATIONAL AUDIT OF MANAGEMENT
}

\section{INTEGRATE (The National ENT Trainee Research Network)}

\author{
Steering Committee: M. Ellis, A. Hall, J. Hardman, N. Mehta, M. E. Smith, R. J. Williams*
}

Executive Committee: S. Carrie, C. Hopkins

Statisticians: J. Chynoweth, B. G. Jones, K Stevens

Site Leads: Y. Abbas, Southend Hospital; M. Abdelkader, Basildon and Thurrock Hospital; M. Adams, Altnagelvin; A. Addison, James Paget Hospital; R. Advani, North Manchester General Hospital; T. Ahmed, Dorset County Hospital; V. Alexander, Brighton General Hospital; V. Alexander, Royal Sussex County Hospital; B. Alli, Bradford Royal Infirmary; S. Alvi, Leighton Hospital; N. Amiraraghi, Crosshouse Hospital; A. Ashman, The Great Western Hospital; R. Balakumar, Gloucestershire Royal Hospital; J. Bewick, Addenbrookes Hospital; D. Bhasker, Pinderfields General Hospital; S. Bola, Wexham Park Hospital; P. Bowles, Worthing Hospital; N. Can Guru Naidu, Barnet Hospital; N. Caton, East Surrey Hospital; J. Chapman, Salisbury District Hospital; G. Chawdhary, Milton Keynes General Hospital; M. Cherko, John Radcliffe Hospital; M. Coates, Sunderland Royal Hospital; K. Conroy, Wythenshawe Hospital, Macclesfield District General Hospital; P. Coyle, Peterborough City Hospital; O. Cozar, University Hospital of North Staffordshire; M. Cresswell, Derriford Hospital; L. Dalton, Arrowe Park; J. Danino, New Cross Hospital; C. Daultrey, Worcester Royal Hospital; K. Davies, Morriston Hospital; K. Davies, Royal Liverpool University Hospital; D. Dick, Royal Victoria Hospital; P. Dimitriadis, Royal Hallamshire Hospital; N. Doddi, Princess of Wales Hospital; M. Dowling, Stepping Hill Hospital; R. Easto, Monklands Hospital; r. edmiston, Royal Albert Edward Infirmary; D. Ellul, Western General Hospital; S. Erskine, West Suffolk Hospital; A. Evans, Barnsley District General Hospital; A. Farboud, University Hospital of Wales; C. Forde, King George Hospital; J. Fussey, Walsall Manor Hospital; A. Gaunt, Queens Medical Centre; J. Gilchrist, Royal Bolton Hospital; R. Gohil, New Royal Infirmary of Edinburgh; E. Gosnell, Royal Blackburn Hospital; D. Grech Marguerat, Royal Cornwall Hospital; R. Green, Ninewells Hospital; R. Grounds, Medway Hospital; A. Hall, Royal National TNE Hospital; J. Hardman, St. Marys Hospital; A. Harris, Royal Gwent Hospital; L. Harrison, Northampton General Hospital; R. Hone, Royal Surrey County Hospital; E. Hoskison, University Hospital of Coventry \& Warwickshire; J. Howard, Wrexham Maelor Hospital; D. loannidis, Royal Hampshire County Hospital; I. Iqbal, Freeman Hospital; N. Janjua, Queen Alexandra Hospital; k. jolly, Russells Hall Hospital; S. Kamal, Poole Hospital; T. Kanzara, Countess of Chester; N. Keates, Torbay Hospital; A. Kelly, Antrim Area Hospital; H. Khan, Fairfield General Hospital; T. Korampalli, Rotherham District General Hospital; M. Kuet, Colchester General Hospital; P. Kulloo, Royal London Hospitals; R. Lakhani, St Georges Hospital; A. Lambert, Charing Cross Hospital; C. Leonard, Royal Belfast Hospital for Sick Children; G. Lloyd, Guys hospital; E. Lowe, Southampton General Hospital; J. Mair, Birmingham Heartlands Hospital; E. Maughan, University College Hospital London; T. Mayberry, Queen Elizabeth Hospital - University Hospitals Birmingham NHSFT; L. McCadden, Craigavon Area Hospital; F. McClenaghan, West Middlesex University Hospital; G. McKenzie, Hull Royal Infirmary, Castle Hill Hospital; R. Mcleod, West Wales Hospital; S. Meghji, Norfolk \& Norwich University Hospital; M. Mian, Furness General Hospital; A. Millington, Ipswich Hospital NHS Trust; O. Mirza, Royal Preston Hospital; S. Mistry, Calderdale Royal Hospital; A. Mitchell-Innes, Birmingham Childrens Hospital; E. Molena, Frimley Park Hospital; J. Morris, Royal United Hospital; T. Myuran, Basildon Hospital, Princess Alexandra Hospital; A. Navaratnam, Northwick Park Hospital; E. Noon, Blackpool Victoria Hospital; O. Okonkwo, Salford Royal Hospital; B. Oremule, Royal Lancaster Hospital; L. Pabla, James Cook University Hospital; E. Papesch, Broomfield Hospital; V. Pattni, Bristol Royal Infirmary; V. Puranik, Ysbyty Gwynedd Hospital; R. Roplekar, Raigmore Hospital; E. Ross, Birmingham City Hospital; E. Schechter, Luton \& Dunstable Hospital; A. Senior, Midlands Treatment Centre (Burton); N. Sethi, Leeds General Infirmary; S. Sharma, Central Middlesex Hospital; R. Sharma, St Johns Hospital;

Z. Sherazi, Tameside General Hospital; A. Tahir, Cumberland Infirmary; T. Tikka, Queen Elizabeth University Hospital; O. Tkachuk Hlinicanova, Glan Clwyd Hospital; K. To, Royal Hospital for Sick Children Edin; E. Toll, Royal Devon \& Exeter Hospital; K. Ubayasiri, Royal Derby Hospital; S. Unadkat, Whipps Cross University Hospital; N. Upile, University hospital of Aintree; A. Vijendren, Lister Hospital; H. Walijee, Alder Hey; M. Williams, Darlington Memorial Hospital; G. Wilson, Leicester Royal Infirmary; W. Wong, York District Hospital; G. Wong, North Hampshire Hospital; C. Xie, Princess Royal University Hospital; A. Yao, Manchester Royal Infirmary; H. Zhang, Queens Hospital;

Corresponding author:

Mr Richard Williams

Institute of Naval Medicine,

Crescent Rd,

Alverstoke,

Gosport.

PO12 2DL

Richard.williams8@nhs.net

\section{Conflicts of interest:}

None declared

\section{Key words:}

Epistaxis; assessment; cautery; packing; haematology; surgery.

\section{Funding:}

This national audit was funded by ENTUK. The funding body had no influence over content. 
Background: Epistaxis is a common condition that can be associated with significant morbidity and places a considerable burden on our healthcare system. This national audit of management seeks to assess current practice against newly created consensus recommendations and expand our current evidence base.

Method: Patients that met inclusion criteria at 113 registered sites across the UK, had their management compared with audit standards during a 30-day window. Data was further utilised for explorative analysis.

Results: 1826 cases were uploaded to the database, representing $94 \%$ of all cases that met the inclusion criteria at participating sites. $62 \%$ of patients were successfully treated by ENT clinicians within 24 hours and the 30 -day recurrent presentation rate across the dataset was $13.9 \%$. Significant event analysis revealed an all-cause 30 -day mortality rate of $3.4 \%$.

Conclusion: Audit findings demonstrate a varying alignment with consensus guidance with explorative analysis countering some previously well-established tenets of management.

\section{INTRODUCTION}

The Care Quality Commission highlights 'Good Governance' as a fundamental standard of care. Central to this, clinical audit seeks to assess the quality and safety of this care. Whilst local audit is well established in otorhinolaryngology, there has been a paucity of national centrally-delivered audit within our specialty since the National Prospective Tonsillectomy Audit ${ }^{(1)}$ and the National Audit of Sino-nasal Surgery ${ }^{(2)}$ published in 2005 and 2006 respectively. ENT-UK and the British Rhinological Society (BRS) sought to address this deficiency by challenging INTEGRATE (the National ENT Trainee Research Network) to design and deliver a national audit of management for the hospital treatment of epistaxis.

Epistaxis is the most common acute disorder managed by ENT services in the UK, with around 25,000 acute presentations to NHS hospitals each year (http://www.hscic. gov.uk/ accessed 11/07/17). Despite this high incidence, prior to this initiative there were no nationally accepted guidelines for its management. A pilot audit $^{(3)}$ led by INTEGRATE has confirmed significant variation in existing treatment between hospital trusts.

This audit aims to compare current management against agreed consensus management guidelines ${ }^{(4)}$, identify variation in practice and perform exploratory analysis of the dataset to expand our current 
understanding of this common condition. As a result of this audit, we seek to generate a program of change to deliver improved and standardised evidence-based hospital care of epistaxis across the UK.

\section{METHOD}

Organisation and design: This audit was designed and delivered by INTEGRATE following the creation of a project steering committee comprising of 6 trainees and 2 consultant executive members. One trainee was nominated to chair the steering committee and co-ordinate the audit.

Audit standards: Following a comprehensive and systematic review of the literature ${ }^{(5-9)}$, national consensus recommendations for the hospital management of epistaxis were developed ${ }^{(4)}$. These recommendations were utilised to generate a draft data-collection tool (DCT) for the audit including 30-day outcome data. The draft DCT was adapted in line with lessons learnt from a multi-centre pilot audit ${ }^{(3)}$ before undergoing multi-level scrutiny, initially by the audit steering committee and subsequently by the ENT-UK executive committee. The agreed DCT was then optimised by commissioned statisticians at the Peninsula Clinical Trials Unit (PenCTU) for ease of subsequent analysis following data collection.

Audit period: A 30-day data audit window was identified from midnight on the 7th of November 2016.

Inclusion/Exclusion: During the audit window, patients $\geq 16 y$ rs old presenting emergently, as an unscheduled event with a diagnosis of epistaxis that were subsequently managed by ENT services were included in the audit. This included patients referred from the Emergency Department (ED), primary care or from other specialties within participating units. Patients attending pre-arranged appointments for the management of chronic self-limiting epistaxis were excluded, as well as patients seen and treated by Emergency Department staff without referral to ENT services. Telephone encounters were not included.

Collaborator Engagement: Site-leads were recruited from ENT departments throughout the UK via a network of regional trainee representatives, engagement with the Specialist Advisory Committee and through open advertisement via the Association of Otolaryngologists in Training. Site-leads were given online supporting material to aid in the local delivery of the audit and were invited to attend a launch event prior to the audit window to further clarify the audit process. Throughout local preparation, site-leads were requested to complete an on-line task list detailing their progress to allow the steering committee to maintain a strategic overview of the project. 
Data collection: Cases meeting the inclusion and exclusion criteria were identified prospectively at the point of presentation. Throughout the audit window, ED and ENT practitioners were encouraged to make detailed clinical notes following all epistaxis related clinical encounters in line with good medical practice $^{(10)}$ guidance. Audit proformas were not utilised by treating clinicians to prospectively record clinical information due to concerns regarding their impact on pre-existing standard practice. At the point of discharge audit data was gathered by collaborators from hospital notes, uploaded via a web-based portal and stored in the Data Safe Haven on a secure server hosted at University College London. The server was certificated to the ISO27001 information security standard and conforms to the NHS Information Governance Toolkit. All connections to the Data Safe Haven server via the web portal were secured with 256-bit SHA encryption.

Following closure of the 30-day window a clinical coding search was conducted in all participating units to highlight all epistaxis presentations during the audit period to established whether any cases had been missed. All cases were examined against inclusion/exclusion criteria and then cross referenced with the cases uploaded to the database. Where cases were missed, hospital notes were requested and if possible, added retrospectively to the database. Any cases identified retrospectively were highlighted as such for the purposes of data analysis. 30-day outcome data was gathered for all uploaded cases via note retrieval through local audit departments. Units were also asked to provide trust quoted population at risk data. No identifiable patient data was uploaded to the server, all submissions were anonymised using an audit specific key for each patient devised and stored locally at each site in line with the clinical governance policy at each site.

Management of data submission and quality: The audit steering committee utilised a dedicated online platform (https://www.entintegrate.org) to facilitate communication between collaborators, to rapidly respond to potential problems and to support timely and high-quality data submission. On completion of data submission, steering committee members inspected submitted data for errors, duplication or omissions. Where necessary, site-leads were contacted to remedy identified discrepancies.

Audit and ethical approval: Prior to data collection, an audit proposal was submitted for approval to the audit departments of all participating units, in line with local policy. Caldicott Guardians were contacted at all sites and approval sought for the method of data collection. Although formal patient consent was neither sought nor required, patient information regarding the audit was displayed at all participating sites and individuals invited to be excluded from the audit if they desired. Many evidence gaps were identified during the consensus recommendations process for epistaxis management ${ }^{(\text {ref })}$. Therefore, we sought NHS Research Ethics Committee (NREC) guidance regarding the use of the dataset beyond comparison against 
identified audit standards. Completion of the Health Research Authority Guidance Tool confirmed formal NREC approval was not required for this purpose.

Statistical Analysis Strategy: When presenting our analysis, we define length of stay (LOS) as the time from hospital presentation to discharge, which reflects the entire treatment process. We define time to ENT as the time from presentation through to first ENT review. Haemostasis time (HT) is the time from first ENT review to when final haemostasis is achieved prior to discharge. A limitation of the data set is that it is unknown whether the patient was actively bleeding on arrival to ENT, however, all patients had been referred for emergency ENT input. We define discharge time as the time from final haemostasis to discharge. Each interval was calculated by gathering data on the absolute date and time of each event. Additionally, we will discuss rates of 30-day recurrent presentation (RP), defined as representations to hospital with epistaxis in the 30 days following the point of initial presentation. The objective of the statistical analysis was primarily to assess compliance with national consensus recommendations that formed our audit standards, and thereafter, to identify which patient factors and treatments (both prior to ENT and at the first ENT review) were associated with variation in outcome in terms of HT and RP.

During the exploratory analysis, it was determined that HT was positively skewed and multimodal as there were two modes (i.e. peaks in the data). To compensate for the skewed distribution of the data, a log transformation was applied to HT. Plots of HT are presented in log hours, but summary statistics are given in hours unless otherwise stated. Although this provides visual representation to observed differences in treatment, it was unable to account for all potential confounding factors. A future study will conduct inferential analysis of the dataset via regression modelling, which will provide us with the tools to account for associations with multiple treatments and potential confounders.

\section{RESULTS}

The audit was conducted simultaneously in 113 hospitals from all regions of the UK serving a combined population at risk of 51 million people. 1826 cases were uploaded, of which 1358 were gathered prospectively, in total this represented $94 \%$ of all cases presenting to involved units that met inclusion criteria. The median number of cases uploaded per unit was 14 (range 1-50). Scrutiny of the dataset discovered 89 cases did not meet inclusion criteria and 157 cases did not report a HT and so were excluded from analysis. Follow-up data was available for 1469 patients who could be matched with the audit data. Once excluding those not within the audit period and undertaking relevant data manipulation, we were left with 1152 patients. 30 patients had a HT of 0 suggesting ED treatment had successfully treated patients without ENT input. Consequently, these patients were excluded leaving 1122 patients for 
analysis. The RP rate for these patients was $24.1 \%$, compared with $13.9 \%$ for the larger group of 1122 patients. Whilst the difference appears substantial, any conclusions drawn from only 30 patients should be treated cautiously.

The median age was 73 years, the inter-quartile range (IQR) was 62-82 years and the range was 16 - 100 years. with larger proportion of males versus females (56\% vs 44\%). The median LOS was 29.5 hours with IQR of 11.1-51.0 hours. This was divided into time to first ENT review (median 2.4, IQR 1.1-4.6), HT (median 18.5, IQR 1.0-33.7) and discharge time (median 3.5, IQR 1.1-17.0). 25\% of patients achieved final haemostasis within 1 hour of their ENT review. Approximately $62 \%$ of cases were successfully treated by ENT within 24 hours of their first review. The longest period for any case to achieve achieved final haemostasis was just over 6 days, with less than $1.5 \%$ of patients requiring treatment for more than 4 days (fig.1). The 30-day RP rate across the data set was 13.9\%. 30-day serious adverse event analysis revealed specific events occurred at the following rates: all-cause mortality $3.4 \%$; myocardial infarction $0.7 \%$; cerebrovascular accident $0.5 \%$; pulmonary embolism $0.2 \%$ and deep vein thrombosis $0.1 \%$.

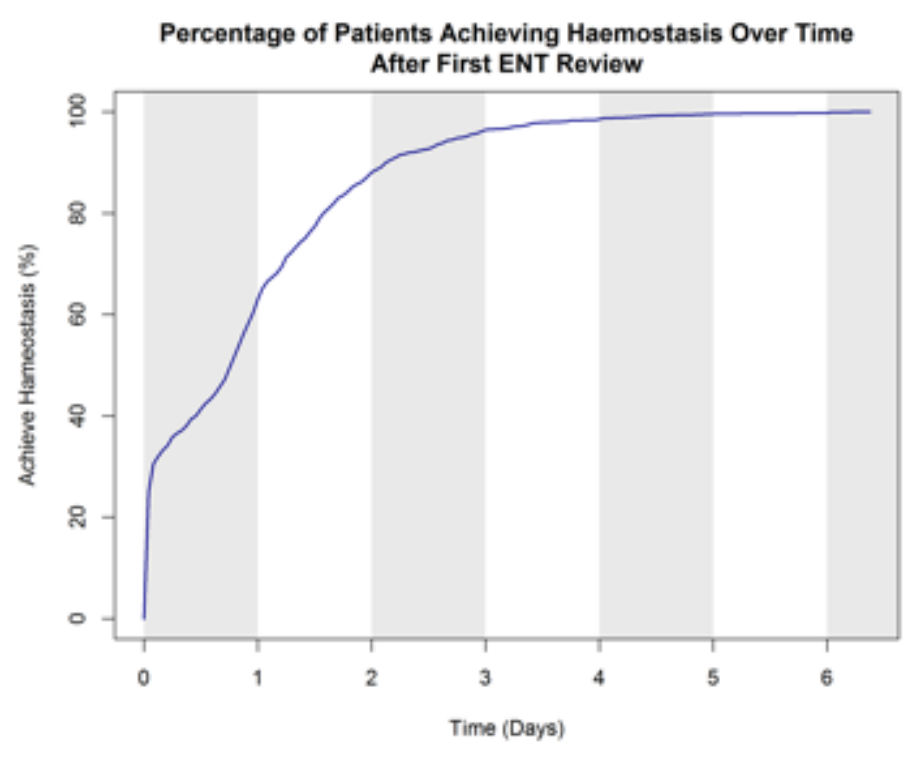

fig. 1 - Patients achieving final haemostasis over time

\section{Initial Assessment:}

The Modified Early Warning Score (MEWS) ${ }^{(11)}$ is a nationally recognised and validated method of patient assessment which combines physiological parameters and observations to rapidly grade a patient's degree of illness. MEWS was reported in 841 cases (75.0\%). Higher MEWS was associated with increased HT; this was evident in the $7.0 \%$ of patients with MEWS $\geq 4$ vs MEWS < 4 (23.0 (IQR 12.7-36.0) vs 18.8 (IQR 1.134.3)) which represented $7.0 \%$ of cases. Patients with MEWS $\geq 4$ were associated with a lower risk of RP compared to patients with MEWS < 4 (0.0\% vs $14.6 \%)$, however, numbers for analysis were small (fig. 2.). 


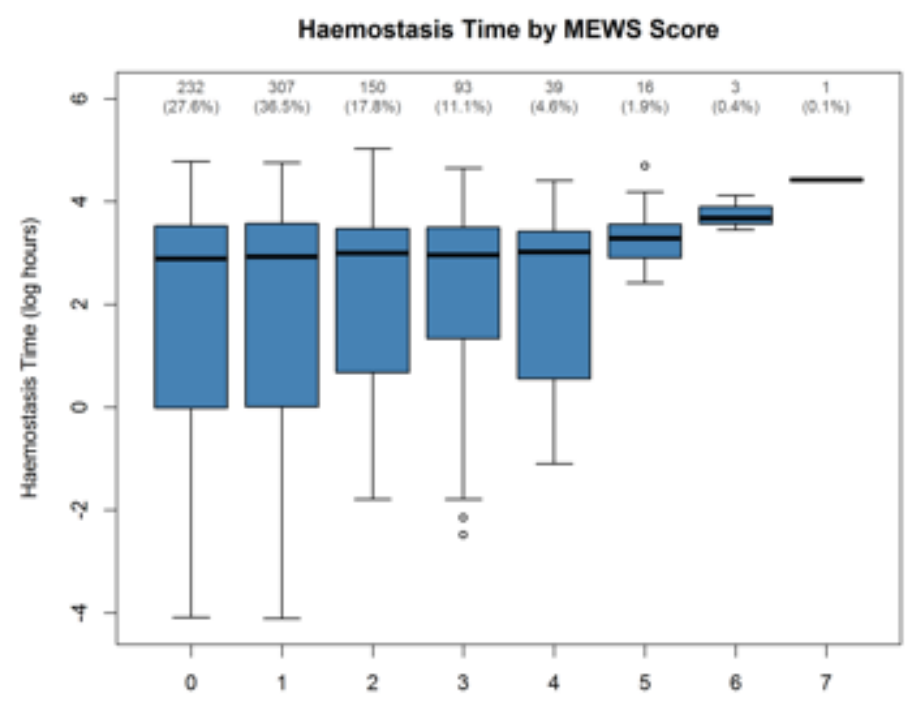

fig. 2-Haemostasis time by MEWS score

The World Health Organisation (WHO) bleeding classification ${ }^{(12)}$ is an internationally accepted method for categorising the severity of bleeding, initially developed in the context of oncology patients. There are various condition-specific sub-classifications including a three-grade score for the severity of epistaxis. This score is calculated according to the total duration of bleeding in the previous 24 hours $(<30 \mathrm{mins}=$ grade I, $>30$ mins II) plus the requirement for transfusion of red blood cells (grade III). This is the only accepted classification for the severity and duration of epistaxis. Within our dataset a WHO grade could be calculated in 1115 cases (99.4\%), with $12.8 \%$ of cases classified grade I, $82.7 \%$ grade II and $4.5 \%$ grade III. Patients with a higher WHO grade had longer median HT, with the largest difference in median between grade I and III (1.0 (IQR 0.3-20.7) vs 42.6 (IQR 19.1-75.8)) (fig. 3). 25.0\% of patients with grade III bleeds represented within 30 -days, compared with $13.5 \%$ of grade II and $12.3 \%$ of grade I.

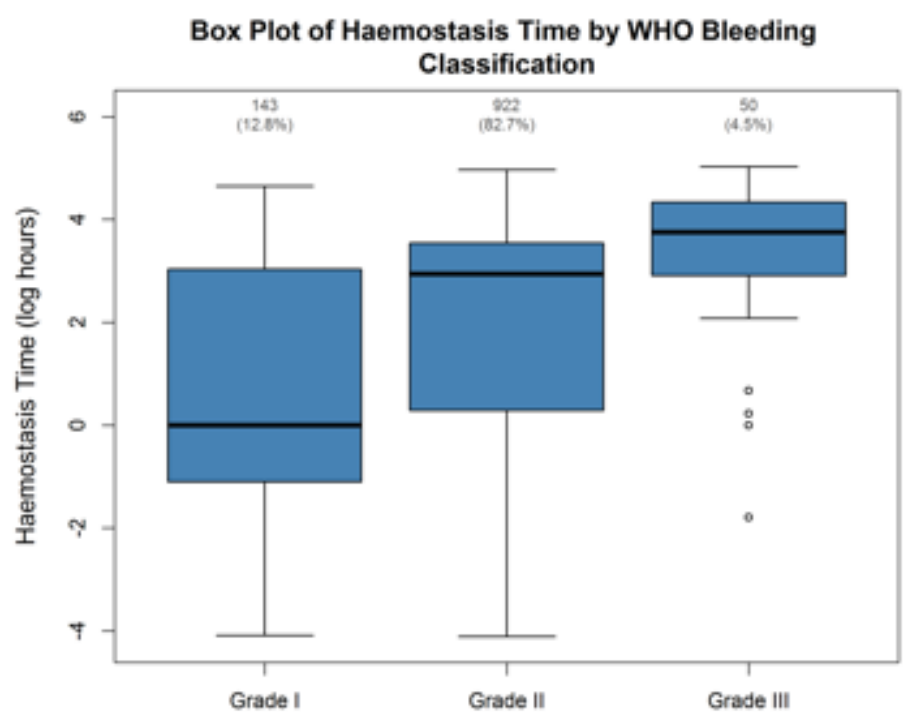

fig. 3-Haemostasis time by WHO bleeding grade 
The initial ENT assessment was predominantly performed by junior-grade doctors ( $n=950,86.6 \%$ ) followed by middle-grade doctors ( $n=107,9.2 \%)$, nurse practitioners $(n=38,3.5 \%)$ and consultants $(n=8,0.7 \%)($ tab. 1). For each WHO bleed grade, specialist nurses have the lowest median HT. However, the number of patients seen by specialist nurses and consultants was very small. Therefore, it is unlikely that the estimates for median HT are representative or reliable. Neither a nurse specialist nor consultant saw a patient with a MEWS $>4,95 \%$ of these patients were attended to by junior grade doctors. Although the evidence suggested the nurses tended to have a lower $\mathrm{HT}$, the 30-day recurrent rates were notably higher. RP for patients seen by nurse practitioners was $21.6 \%$, compared to junior-grades with $13.6 \%$, middlegrades with $14.6 \%$ and consultants with $14.3 \%$.

\begin{tabular}{|c|c|c|c|c|c|c|}
\hline \multirow{2}{*}{$\begin{array}{l}\text { Practitioner } \\
\text { seniority }\end{array}$} & \multicolumn{2}{|l|}{ WHO I } & \multicolumn{2}{|l|}{ WHO II } & \multicolumn{2}{|l|}{ WHO III } \\
\hline & Median (IQR) & $\mathbf{n}$ & Median (IQR) & $\mathbf{n}$ & Median (IQR) & $\mathbf{n}$ \\
\hline Consultant & 0.3 & 1 & $5.5(1.4-25.0)$ & 7 & - & 0 \\
\hline Junior grade & $1.0(0.3-20.3)$ & 122 & $19.2(2.0-34.7)$ & 781 & $42.6(22.4,72.1)$ & 42 \\
\hline Middle grade & $3.5(1.5-20.3)$ & 9 & $23.8(1.4-37.2)$ & 84 & $75.9(31.6-81.2)$ & 6 \\
\hline Nurse specialist & $0.7(0.5-5.3)$ & 6 & $1.0(0.4-26.7)$ & 31 & 8.0 & 1 \\
\hline
\end{tabular}

Tab.1 Median homeostasis time in hours (IQR) grouped by severity of bleeding and seniority of practitioner

Regarding co-morbidities, a past medical history of hypertension (formally diagnosed through sustained ambulatory blood pressure monitoring) was reported in 55\% of cases. The median HT in these patients was 20.3 (IQR 1.5-36.0) compared with 15.2 in those without a history of hypertension (IQR 0.6-29.7) and RP rates were $14.0 \%$ vs $13.8 \%$ respectively. $26.0 \%$ of cases reported previous epistaxis presentations with in the preceding year. Median HT was 21.3 (IQR 1.3-37.2) in patients declaring previous bleeds vs 17.7 (IQR 0.9-31.3) who did not, RP was nearly double in these patients (20.2\% vs $12.1 \%$ ). Patients who subsequently represented within 30-days of discharge had a shorter median HT during their initial treatment (16.5 vs 18.6). 156 (14.4\%) individuals had a past medical history of diabetes mellitus and 333 (30.4\%) had ischaemic heart disease. However, neither condition demonstrated a potential association with varying haemostasis time. The RP was greater in those with a history of diabetes mellitus $(21.4 \%$ vs $12.3 \%)$ and also greater in those with a history of ischaemic heart disease (14.9\% vs $13.0 \%)$.

A history of previous epistaxis presentation within the preceding year was reported in $26.0 \%$ of cases. Patients declaring previous bleeds had a longer median HT of 21.3 (IQR 1.3-37.2) compared to 17.7 (IQR 0.9-31.3) for those who did not. The RP was also greater in these patients at $20.2 \%$ compared to $12.1 \%$. In the subset of patients who subsequently represented with a second episode of epistaxis within 30-days of their primary presentation, the subsequent median HT was found to be longer (16.5 vs 18.6 hours).

Data were collected regarding time of presentation to investigate any associations with out of hours treatment. Working hours were defined as 0800-1700 and out of hours were defined as 1700-0800, seven 
days a week. There was no potential association between median HT and day of presentation or whether presentation was in-hours or out-of-hours. Anterior rhinoscopy was performed at initial assessment in $71.9 \%$ of cases. Analysis of the outcome of these patients demonstrated no difference in median HT or RP of $13.6 \%$ when compared to patients who did not undergo this examination. A full blood count (FBC) was performed in $84.0 \%$ of cases and a coagulation screen in $65.4 \%$. Patients who underwent these investigations had longer median HT when compared to patients who did not have the specific test (FBC 20.4 vs 0.8 , coagulation screen -20.5 vs 9.4 )

\section{Cautery:}

Cautery was performed in 365 cases (32.5\%) during the initial ENT review, with a topical vasoconstrictor used in $116(31.8 \%)$ of cases. Silver nitrate was utilised in most cases (97.5\%) with only 9 patients treated with bipolar cautery. Median HT was 0.5 hours in the silver nitrate group (IQR = 0.3-2.9), however, low numbers in the bipolar group made meaningful comparison impossible. Patients who received cautery at their first ENT review had a substantially shorter median HT (fig. 4). 30-day outcome data demonstrated a higher RP in patients who underwent cautery during their first ENT review (17.3\% vs 12.3\%). Rigid endoscopy or microscopy was utilised during cautery at the initial ENT review in 17 (4.7\%) cases.

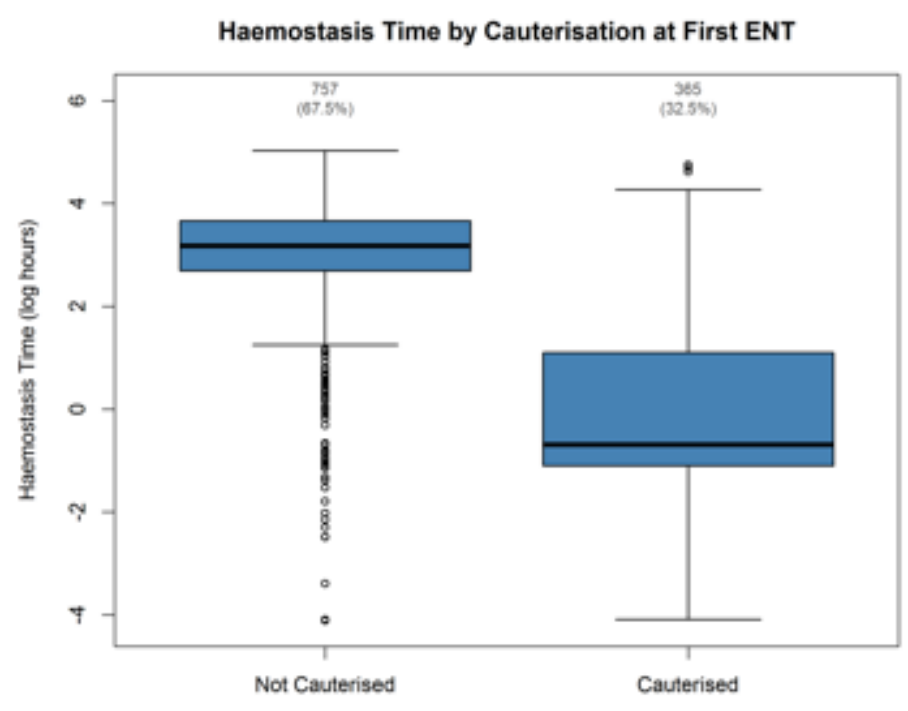

fig. 4 - Haemostasis time by cautery status

Intranasal devices and haemostatic agents:

In total, 517 (46.1\%) patients were packed prior to ENT review. Packing prior to ENT review was associated with a longer median haemostasis time, however, if this pre-ENT pack was removed during initial ENT assessment then haemostasis time reduced substantially. In fact, there was minimal difference in haemostasis time between those who were never packed compared with those who had their pre-ENT pack removed at initial ENT assessment with no subsequent replacement packing (fig. 5). Patients packed 
prior to ENT review and patients packed at initial ENT review had a lower RP when compared with patients who were never packed (packed prior to ENT $=12.8 \%$ vs $14.9 \%$, packed by ENT $=9.4 \%$ vs. $15.5 \%) .49(6.4 \%)$ of patients who were packed received antibiotics upon discharge.

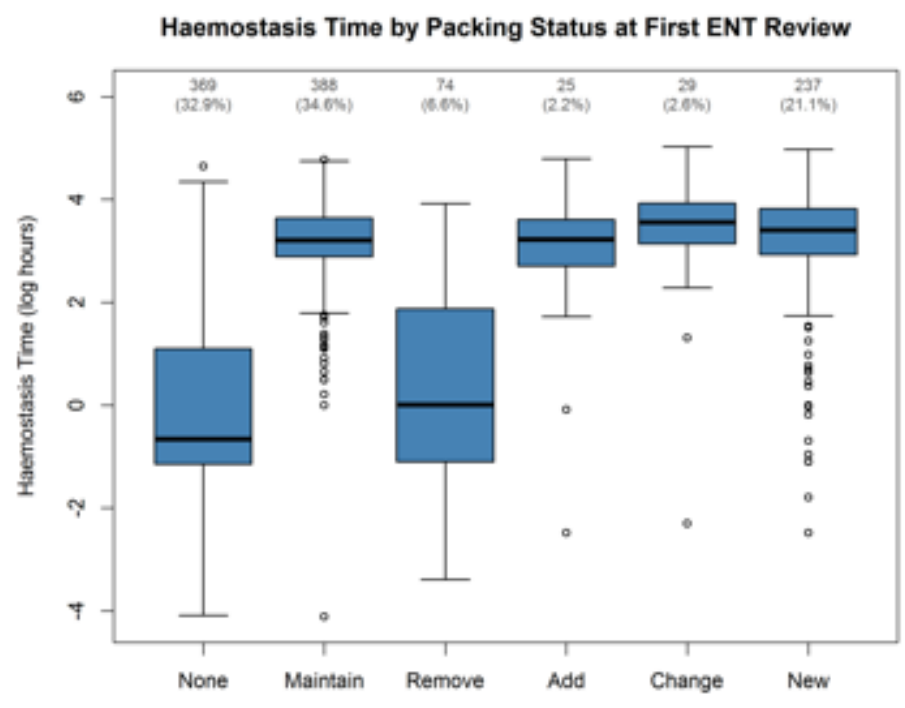

fig. 5 - Haemostasis time by packing status

The type of packing used was classified into one of five categories: inflatable (e.g. Rapid rhino), nondissolvable (e.g. Merocel), dissolvable (e.g. Nasopore), urinary catheter or haemostatic agent (e.g. Kaltostat). $75.4 \%$ of the patients packed prior to ENT received an inflatable packing (tab. 2). The median and inter quartile range for HT by pack type shows no difference between inflatable and non-dissolvable packs used prior to and by ENT. The number of patients packed with dissolvable, catheter and haemostatic packs was too small to gain useful insight.

\begin{tabular}{|c|c|c|c|c|c|}
\hline Consultation & Pack type & n (\%) & Median HT (IQR) & & \\
\hline \multirow{2}{*}{$\begin{array}{l}\text { Prior to first } \\
\text { ENT review }\end{array}$} & Inflatable & $390(74.7)$ & 23.4 & $(150,36.9)$ & $14.1 \%$ \\
\hline & Non dissolvable & $128(24.5)$ & 23.3 & $(15.7,37.9)$ & $9.1 \%$ \\
\hline \multirow{4}{*}{$\begin{array}{l}\text { At first ENT } \\
\text { review }\end{array}$} & Inflatable & $250(83.3)$ & 30.6 & $(20.0,46.1)$ & $8.2 \%$ \\
\hline & Non-dissolvable & $32(10.7)$ & 28.8 & $(19.5,47.5)$ & $6.9 \%$ \\
\hline & Dissolvable & $14(4.7)$ & 11.3 & $(1.1,29.4)$ & $33.3 \%$ \\
\hline & Catheter & $4(1.3)$ & 41.3 & $(16.4,75.5)$ & $25.0 \%$ \\
\hline
\end{tabular}

Tab. 2 Type of packs used and HT. Prior to the first ENT review there were also cases with dissolvable packs ( $n=2)$, catheter ( $n=1)$ and haemostatic agent ( $n=1)$.

Of those packed with inflatable or non-dissolvable packs, the median number of packs placed per patient was 1 (IQR 1-2, range 1-6). Patients requiring multiple packs were associated with an HT of 32.3 (IQR 20.348.5) versus those with a single pack of 21.5 (IQR 12.5-34.5) and a RP of $9.7 \%$ vs $13.8 \%$. Cautery was performed in 295 (38.8\%) of packed patients following removal of their final pack. The RP was lower in this 
cautery group at $10.9 \%$ versus $12.9 \%$ amongst those packed but not cauterized following removal. The median time to discharge from removal of final pack was 4.1 hours (IQR 1.5-16.0 hours).

\section{Haematological factors:}

In total 572 (51.0\%) of the patients were on some form of antithrombotic medication (tab.3). Patients not on antithrombotic medication had a shorter median HT than those on anti-thrombotic medication (17.0 (IQR 0.9-32.9) vs 19.3 (IQR 1.3-34.7)). Patients whose medication was stopped or reversed had a prolonged median HT compared to those who continued some form of treatment, this was the case across all medication types. The cessation of antiplatelet agents was associated with lower RP as was the cessation or reversal of warfarin, however, this was not seen when DOACs were withheld. The International Normalised Ratio (INR) of patients who had their warfarin reversed was higher than any other group of patients (tab. 4). Median HT for patients taking warfarin was longer than patients taking DOACs. The median HT for patients on warfarin was 23.3 (IQR 4.9-39.0) vs oral anticoagulants 18.4 (IQR 1.6-29.0). The RP for all patients taking warfarin compared with oral anticoagulants was similar at $14.9 \%$ vs. $14.3 \%$.

\begin{tabular}{|c|c|c|c|c|c|}
\hline \multirow{4}{*}{\begin{tabular}{|l|} 
Medicaton \\
Aspirin
\end{tabular}} & \multirow{4}{*}{\begin{tabular}{|l}
$\begin{array}{c}\text { Status during } \\
\text { admission }\end{array}$ \\
Unchanged \\
Stopped or \\
Altered
\end{tabular}} & \multirow{2}{*}{$\begin{array}{c}\text { n (\%) } \\
157(14.0)\end{array}$} & \multicolumn{2}{|c|}{ Median HT (IQR) } & \multirow{2}{*}{$\begin{array}{r}\text { RP (\%) } \\
11.7\end{array}$} \\
\hline & & & 12.5 & $(0.4,26.0)$ & \\
\hline & & $53(4.7)$ & 25.1 & $(17.5,36.5)$ & 6.5 \\
\hline & & $1(0.1)$ & & & \\
\hline \multirow[t]{3}{*}{ Clopidogrel } & Unchanged & $67(6.0)$ & 17.5 & $(0.4,38.0)$ & 14.5 \\
\hline & Stopped & $33(3.0)$ & 25.2 & $(16.5,38.3)$ & 9.7 \\
\hline & Altered & $2(0.1)$ & & & \\
\hline \multirow[t]{3}{*}{ Heparins } & Unchanged & $18(1.6)$ & 2.8 & $(0.5,26.6)$ & 18.8 \\
\hline & Stopped & $6(0.5)$ & 44.1 & $(20.0,50.6)$ & 0.0 \\
\hline & Altered & $2(0.2)$ & & & \\
\hline \multirow[t]{3}{*}{ DOAC } & Unchanged & $65(5.8)$ & 2.9 & $(0.5,21.3)$ & 12.3 \\
\hline & Stopped & $74(6.6)$ & 23.4 & $(13.7,37.9)$ & 14.5 \\
\hline & Altered & $3(0.3)$ & 20.8 & $(10.6,35.1)$ & 33.3 \\
\hline \multirow[t]{4}{*}{ Warfarin } & Unchanged & $85(7.1)$ & 11.0 & $(0.4,27.3)$ & 24.3 \\
\hline & Stopped & $105(9.4)$ & 28.8 & $(18.0,42.4)$ & 9.8 \\
\hline & Reversed & $16(1.4)$ & 36.9 & $(23.8,49.4)$ & 0.0 \\
\hline & Altered & $11(1.0)$ & 19.0 & $(0.9,33.6)$ & 12.5 \\
\hline
\end{tabular}




\begin{tabular}{|l|c|cc|}
\hline \multicolumn{1}{|c|}{ Status during admission } & N (\%) & Median INR & IQR \\
\hline Unchanged & $67(5.9)$ & 2.6 & $(2.1,2.9)$ \\
\hline Stopped & $93(8.3)$ & 2.7 & $(2.4,3.2)$ \\
\hline Reversed & $15(1.3)$ & 3.9 & $(3.2,6.6)$ \\
\hline Altered dose & $10(0.9)$ & 3.0 & $(2.6,3.6)$ \\
\hline
\end{tabular}

Tab. 4 Warfarin status by INR

Tranexamic acid (TXA) was administered to 92 (8.2\%) of patients. The box plots suggest a marginally longer median HT for patients receiving TXA (fig. 6). RP rates were also higher in patients who received TXA during their treatment (18.1\% vs $13.5 \%$ ). MEWS score data demonstrates patients receiving TXA tended to possess a higher degree of illness (tab. 5). Data suggests a slight increase in the time taken to achieve haemostasis in those receiving TXA via IV versus PO, and another slight increase between IV and Topically. However, these differences are relatively small. Furthermore, the amount of data available when split by mode of administration is small, and as such any results should be interpreted with caution. Transfusion of blood products was performed in 50 (4.5\%) cases. Patients transfused had a median pre-transfusion haemoglobin of 91g/L (IQR 7-103) and a median MEWS of 1 with IQR $(1,2)$.
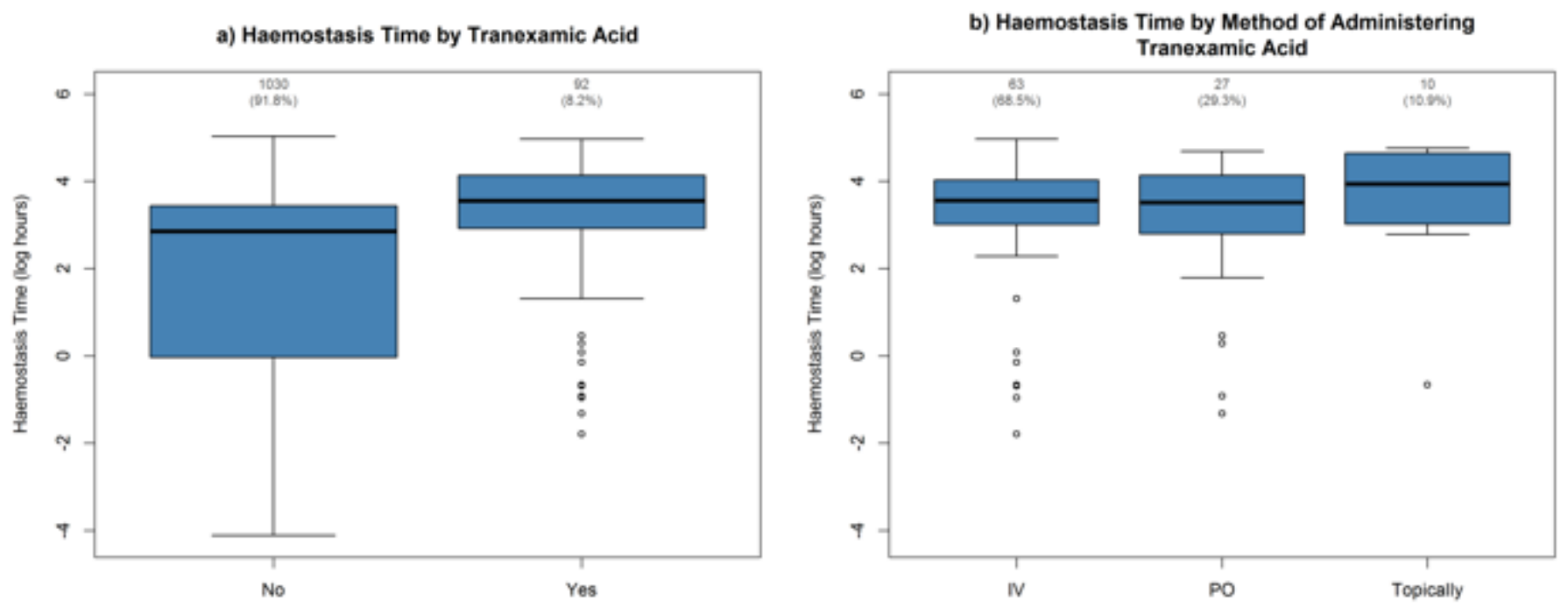

Fig. 6 - Haemostasis Time by tranexamic acid use and method of administration

\begin{tabular}{|l|r|r|}
\hline \multirow{2}{*}{ MEWS } & \multicolumn{2}{|c|}{ Tranexamic Acid } \\
\cline { 2 - 3 } & N(\%) \\
\hline 0 & $216(28.0)$ & Yes \\
1 & $383(36.7)$ & $24(34.8)$ \\
2 & $140(18.1)$ & $10(14.5)$ \\
3 & $85(11.0)$ & $8(11.6)$ \\
4 & $32(4.1)$ & $7(10.1)$ \\
5 & $14(1.8)$ & $2(2.9)$ \\
6 & $1(0.1)$ & $2(2)$. \\
7 & $1(0.1)$ & - \\
\hline
\end{tabular}


Surgery and Radiological Intervention:

$36(3.2 \%)$ of included patients underwent surgery for epistaxis during the audit period. The median time between first ENT review and surgery was 24.7 and IQR $(8.9,54.2)$. Prior to surgery, these patients had a median number of pre-op non-dissolvable pack (inflatable + non-dissolvable) placements of 1 (IQR 1-2, range 0-6). Patients undergoing surgery had a median MEWS of 1 and WHO grade of 2, this was the same as patients in the non-surgery group. Data were available on the grade of principal surgeon grade for 34 of the 36 patients with consultants operating most commonly (52.9\%) followed by registrars (44.1\%). Operations performed were sphenopalatine artery ligation $(n=19)$, sphenopalatine artery cautery $(n=10)$, electrocautery of bleeding point $(n=8)$, septoplasty $(n=4)$, anterior ethmoid artery ligation $(n=3)$ and antrostomy $(n=1)$ with multiple operations being performed in some cases. The number of patients achieving final haemostasis at time of surgery was $17(47.2 \%)$ and the RP rate of surgical patients was 22.6\%. 3 patients underwent radiological intervention with the low numbers preventing detailed analysis. All underwent intervention 2 days following first ENT review. The maxillary artery was embolised in 2 cases and isolated sphenopalatine artery embolisation was performed in 1 case.

\section{DISCUSSION \& LIMITATIONS}

This large multicentre audit sought to benchmark current epistaxis management, enhance the evidencebase and showcase trainee collaborative research as a strategy for large volume data collection. The dataset successfully captured the vast majority of epistaxis presentations within involved units. Data quality in general was high, however, it was limited by reliance on the detailed note taking of clinicians. It remains unclear whether missing data were due to deficiencies in management, note taking or data collection. Incomplete data necessitated the exclusion of a number of cases from analysis, which had otherwise met the audit inclusion criteria. This explorative analysis was able to identify potential associations between patient factors, treatment factors and outcomes. Further inferential statistical analysis is planned, in a subsequent paper, to correct for confounding variables and modelling and identify optimal treatment strategies. Without this additional analysis, suggested associations noted within the dataset thus far should be interpreted with caution.

The large number of cases identified in this study highlights the considerable burden epistaxis places on our healthcare system, both at initial presentation and in the $13.9 \%$ of cases which represented to hospital attendance. The majority of cases were successfully treated within $24 \mathrm{hrs}$, suggesting a limited impact of disease on these individuals. However significant event analysis revealed a 3.4\% 30-day all-cause mortality 
rate within our dataset. In context, the equivalent 30-day all-cause mortality rate for hip fracture in the UK is 7.1\% ( http://www.nhfd.co.uk/nhfd/nhfd2015reportPR1.pdf/ accessed 11/07/2017), a figure that has reduced from $10.9 \%$ over 8 years since the introduction of a program of ongoing national audit. Whilst such figures should be interpreted with caution, and it is acknowledged that epistaxis may not be the condition directly leading to death in these cases, it highlights the level of morbidity held amongst the population of patients we treat.

Assessment of auditable standards within the domain of initial assessment demonstrated high levels of adherence for the recording of key elements of the history, evidenced to effect outcome, within the medical notes. Interestingly, our dataset supported a marked impact of some of these factors on outcome (duration of epistaxis, previous epistaxis, diabetes mellitus), whereas conditions such as ischaemic heart disease and known hypertension seemed to have minimal effect. Initial ENT assessment was most frequently performed by junior doctors, however, reassuringly outcomes associated with these assessments were similar to those of their senior colleagues when cases were analysed by bleed severity. Anterior rhinoscopy was performed commonly and the majority of patients were investigated with a FBC and coagulation screen. Cases where no blood tests were performed experienced a much shorter median $\mathrm{HT}$. This may suggest a lower complexity of presentation and, therefore, that investigations were ordered selectively on the basis of clinical concern, as the consensus statement recommends. Audit data allowed us to calculate a WHO bleed severity in nearly all cases, however, the utility of this relatively crude grading system is questionable.

At the time of initial ENT assessment, less than a third of patients had intranasal cautery performed and similarly only a third of patients had a topical vasoconstrictor applied. Both of these techniques have been recommended as first line treatment strategies in the consensus document. The use of electrocautery as first line was vanishingly rare despite its potentially superior outcomes ${ }^{(13)}$. Surprisingly, despite the dataset showing a lower median HT for those undergoing cautery, the recurrent bleed rate was nearly $50 \%$ higher, contrary to evidence previously published ${ }^{(14)}$. The majority of cautery was performed by junior doctors, though there was little to suggest that outcomes differed between clinician group.

Nearly half of all patients referred to ENT were packed by other specialties prior to assessment, with the indication for such packs unclear from the dataset. Packed patients were associated with longer median $\mathrm{HT}$, but interestingly the small population who had their packs removed at initial assessment experienced a median HT that was the equivalent to the unpacked cases. This supports the treatment strategy of pack removal at the point of initial ENT review to enable a more definitive assessment. The lower RP seen in patients who were packed during their treatment suggests that such devices may not only be effective at 
achieving temporary haemostasis, but that they may also have a favourable effect on longer term outcomes. Over three-quarters of patients receiving intranasal packs had an inflatable device used, as favoured in the consensus recommendations. The median HT was similar for inflatable and non-dissolvable packs, however, RP was lower for the latter group. Following final pack removal, the median discharge time was found to be in line with the consensus recommendations, however, less than half of these patients underwent the recommended post-pack cautery. Cautery following final pack removal was associated with a modestly lower RP.

Over half of all patients were taking antithrombotic medication at initial presentation. As per the consensus recommendations, the majority of patients continued to take antiplatelet medication throughout their treatment. Those who stopped, had a lengthier HT which may suggest a greater burden of disease, although this group had a RP that was nearly half of that for patients continuing antiplatelet therapy. However, the number of representations was small, and a more detailed consideration of the risk and benefits of halting antiplatelet therapy for short periods would need to be explored.

Warfarin and DOACs were the most common anticoagulants taken by epistaxis patients, with treatment stopped or reversed more commonly than maintained. Contrary to consensus recommendations, the groups of patients who had warfarin therapy stopped or maintained both had similar median INR value, both within the most common therapeutic window of 2.0-3.0. However, the dataset shows a much lower RP in patients who have warfarin stopped during their admission, suggesting such practice has a beneficial effect on epistaxis-specific outcome. Patients who had warfarin reversed had a notably higher median INR, suggesting an element of patient selection in these cases. Epistaxis-specific outcomes between the warfarin and DOAC groups were similar, which reassures us of the relative safety of these novel agents despite current fears relating to the inability to reverse them.

Data on TXA use showed longer median HT and higher RP in patients administered with the medication. However, the severity of illness differed notably in those receiving TXA and may represent a confounding factor. Consequently, conclusions regarding the efficacy of TXA are unclear from this dataset. Current practice appears to be in line with the consensus recommendation supporting the use of TXA in selected patients.

Transfusion of blood products occurred more commonly than previously established in the literature ${ }^{(\text {ref })}$ with a median haemoglobin $(\mathrm{Hb})$ at point of transfusion of $91 \mathrm{~g} / \mathrm{L}$. This is above to $70-90 \mathrm{~g} / \mathrm{l}$ target $\mathrm{Hb}$ recommended in the management of major trauma ${ }^{(15,16)}$ and with $25 \%$ of transfused patients receiving 
blood products with a triggering $\mathrm{Hb}$ of $>103 \mathrm{~g} / \mathrm{L}$ it appears at present that the threshold to transfuse in some units may be too low.

Surgery for epistaxis was performed infrequently, however, with a median time to intervention of just over $24 \mathrm{hrs}$ and on average just one pre-operative pack insertion, escalation to theatre appeared expeditious. Despite this, there were still cases where patients received up to six pre-operative packing episodes prior to surgical intervention. Small numbers limit the ability to draw conclusions regarding the efficacy of such procedures, however, given there was no difference between bleed severity and patient illness level between the surgical and non-surgical groups the RP rate of surgical patients of $22.6 \%$ appears high. It is unclear from the dataset what the specific indications for surgical escalation were, especially in the context of a median pre-operative pack of just 1 and similar MEWS and WHO grade when comparing surgery and non-surgery groups. The use of interventional radiology was very rare.

\section{CONCLUSIONS}

This large multicentre audit of epistaxis management further demonstrates the ability of trainee collaboratives to co-ordinate national projects involving the large-scale collection of data. The dataset demonstrates management practices that variably adhere to the newly formed consensus recommendations. Elements of this explorative analysis question our current understanding of previously well-established tenets of management for this common condition. The observational nature of this study limits the strength of conclusions made, however, these findings highlight a number of areas where further research should be directed.

\section{REFERENCES}

1. Lowe D, van der Meulen J, Cromwell D, Lewsey J, Copley L, Browne J, et al. Key messages from the national prospective tonsillectomy audit. Laryngoscope 2007;117:717-24

2. Hopkins C, Browne JP, Slack R, Lund V, Topham J, Reeves B et al. The national comparative audit of surgery for nasal polyposis and chronic rhinosinusitis. Clin otol 2006;31:390-8

3. Mehta N, Williams RJ, Smith ME, Hall A, Hardman JC, Cheung L, et al. Can trainees design and deliver a national audit of epistaxis management? A pilot of a secure web-based audit tool and research trainee collaboratives. J Laryngol Otol 2017;131:518

4. BRS Consensus recommendations on the management of epistaxis (Submitted J Laryngol Otol)

5. Initial Assessment SR (Submitted J Laryngol Otol)

6. Cautery SR (Submitted J Laryngol Otol)

7. Pack SR (Submitted J Laryngol Otol) 
8. Haematology SR (Submitted J Laryngol Otol)

9. Surgery/IR SR (Submitted J Laryngol Otol)

10. General Medical Council. Good medical practice. London: GMC, 1998

11. Subbe CP, Kruger M, Rutherford P, Gemmel L. Validation of a modified Early Warning Score in medical admissions. Qjm 2001;94:521-6.

12. Miller AB, Hoogstraten B, Staquet M, Winkler A. Reporting results of cancer treatment. Cancer 1981;47:207-14.

13. Soyka MB, Nikolaou G, Rufibach K, Holzmann D. On the effectiveness of treatment options in epistaxis: an analysis of 678 interventions. Rhinology 2011;49:474-8.

14. Henderson $A H$, Larkins $A$, Repanos $C$. The use of bipolar electrocautery in adult epistaxis management: Using audit of one hundred and twenty-four cases to define a standardised protocol. Clin Otol 2013;38:554-8

15. Murer K, Ahmad N, Roth BA, Holzmann D, Soyka MB. THREAT helps to identify epistaxis patients requiring blood transfusions. J Otolaryngol 2013;42:4

16. Pollice PA, Yoder MG. Epistaxis: a retrospective review of hospitalized patients. J Otolaryngol 1997;117:49-53. 